\title{
ON DIFFERENTIAL OPERATORS AND AUTOMORPHIC FORMS
}

\author{
BY \\ H. L. RESNIKOFF(1)
}

1. Let $f(z)$ be an automorphic form of dimension $-k$ belonging to the horocyclic group $\Gamma$ acting on the upper half plane $H=\{x+i y \mid x, y \in R$ and $y>0\}$. R. A. Rankin [3] has found all polynomials (with complex coefficients) in $f$ and its derivatives which are automorphic forms for $\Gamma$. His results are expressed as polynomials in $f$ and certain auxiliary functions $\psi_{m}(f), m=2,3,4, \cdots$ defined by

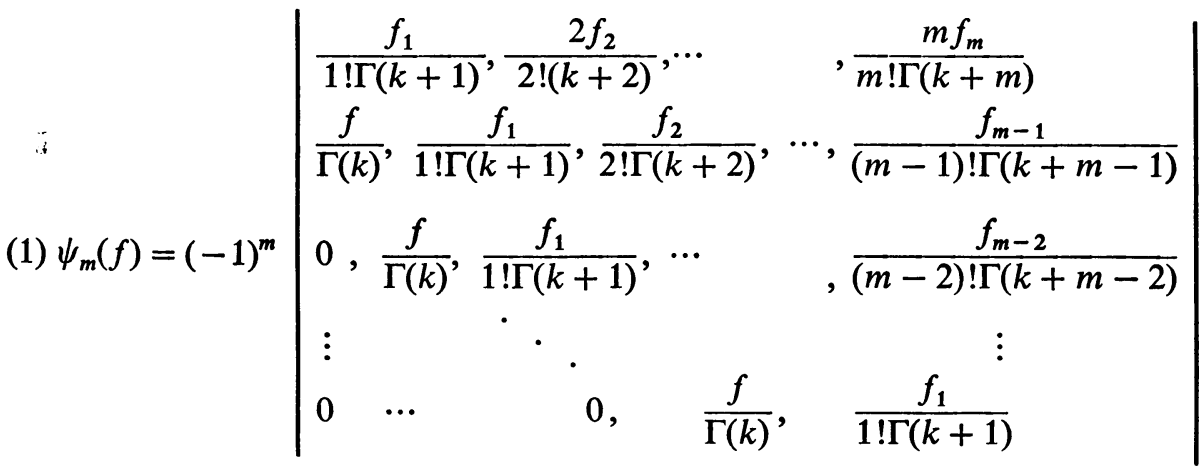

where $f_{n}=d^{n} f / d z^{n}$ and $\Gamma(s)$ is the Euler gamma function. The principal purpose of this note is to provide an alternative presentation of Rankin's main result in terms of another set of auxiliary functions which is often simpler to use, and which can easily be generalized to certain classes of automorphic forms of several complex variables.

2. Denote by $\mathscr{V}(\Gamma, k, v)$ the vector space (over $\boldsymbol{C}$ ) of automorphic forms of dimension $-k$ and multiplier system $v$ for the horocyclic group (Grenzkreisgruppe) $\Gamma$, and by $G(\simeq \operatorname{SL}(2, R)$ modulo its center $)$ the full group of analytic automorphisms of the upper half plane $H$.

If $\mathscr{D}_{z}$ is a polynomial differential operator with coefficients in $C$ which operates with respect to the variable $z$, then $\mathscr{D}_{z}$ maps $\mathscr{V}(\Gamma, k, v)$ into $\mathscr{V}\left(\Gamma, k^{\prime}, v^{\prime}\right)$ for all discrete groups $\Gamma \subset G$ if and only if

$$
\mathscr{D}_{g z}\left(v(g)(c z+d)^{k} f(z)\right)=v^{\prime}(g)(c z+d)^{k^{\prime}} \mathscr{D}_{z} f(z)
$$

Received by the editors September, 1963 and, in revised form, December 13, 1965.

(1) The author is appreciative of support by the U. S. Air Force Office of Scientific Research (AF-AFOSR-359-64). 
for all $g \in G$ (where $g z=(a z+b)(c z+d)^{-1}$ and $\operatorname{det}\left(\begin{array}{l}a b \\ c d\end{array}\right)=1$ ) and all sufficiently differentiable functions $f$. Since $\left\{T_{a} \mid z \rightarrow T_{a} z=z+a, a \in R\right\}$ and $J: z \rightarrow-z^{-1}$ generate $G$, it is enough to have equation(2) satisfied for all $T_{a}, a \in R$, and for $J$.

Let us focus our attention on the linear differential operators $\mathscr{L}_{z}$ with complex coefficients which satisfy equation (2) for all $g \in G . \mathscr{L}_{z}$ must have the form

$$
\mathscr{L}_{z} f(z)=\sum_{m} a_{m} f_{m}(z), \quad a_{m} \in C
$$

For $f \in \mathscr{V}(\Gamma, k, v)$, a straightforward calculation, as in [3], shows that

(4) $f_{m}(g z)=v(g)(c z+d)^{k+2 m} \sum_{l=0}^{m}\left(\begin{array}{c}m \\ l\end{array}\right) \frac{\Gamma(k+m)}{\Gamma(k+m-l)}\left(\frac{c}{c z+d}\right)^{l} f_{m-l}(z)$,

from which it follows, as in [3], that $\mathscr{L}_{z}$ satisfies equation (2) for all $g \in G$ if and only if

$$
k+m-1=0 \text { and } k^{\prime}=m+1,
$$

and in this event, $\mathscr{L}_{z}$ is a (complex) constant multiple of $L^{m} f(z) \equiv_{\mathrm{def}} d^{m} f(z) / d z^{m}$, for some integer $m$ greater than 1 . The equation (2) becomes

$$
L_{g z}^{m}\left(v(g)(c z+d)^{1-m} f(z)\right)=v(g)(c z+d)^{1+m} L_{z}^{m} f(z),
$$

which is equivalent to

$$
\left(\frac{d^{m}}{d z^{m}} \equiv\right) L^{m}: \mathscr{V}(\Gamma, 1-m, v) \rightarrow \mathscr{V}(\Gamma, 1+m, v)
$$

This result is well known; it appears in [1] and [2] as well as implicitly in [3]. There is another, less direct, way of deriving equation (7), which depends on the theory of the Riemann-Liouville operators of fractional integration and differentiation. This method is of special interest because it permits equation (7) to be generalized to the corresponding situation for automorphic forms defined on tubes over exponentials of compact real Jordan algebras, and in particular, to the Siegel upper half planes. To begin, put

$$
I_{a}^{s} f(x)=\frac{1}{\Gamma(s)} \int_{a}^{x} f(u)(x-u)^{s-1} d u
$$

for $f$ continuous on a real interval containing $a$ and $x$, and $s$ a positive real number. It is part of the elementary theory of the Riemann-Liouville integrals (cf. [5]) that $I_{a}^{s} f(x)$ possesses an analytic continuation to the complex s-plane, and furthermore that

$$
I_{a}^{t} \circ I_{a}^{s}=I_{a}^{t+s} \text { for all } s, t \in C,
$$

and $I_{a}^{0}$ is the identity operator on functions. 
LEMMA 1. (i) $I_{a}^{s}$ is independent of $a$ if and only if $s$ is a nonpositive integer. (ii) If $s$ is a negative integer, $s=-m$, then $I_{a}^{s}=d^{m} / d x^{m}$.

Proof.

$$
\begin{aligned}
\frac{d^{m}}{d x^{m}} \circ I_{a}^{s} f(x) & =\frac{1}{\Gamma(s)} \int_{a}^{x} f(u) \frac{\Gamma(s)}{\Gamma(s-m)}(x-u)^{s-m-1} d u \\
& =I_{a}^{s-m} f(x), \quad \text { for } s-m>0 .
\end{aligned}
$$

Analytic continuation of the equation insures its validity for all integers $m$ and $s \in C$. Setting $s=0$ in equation (10) yields

$$
\frac{d^{m}}{d x^{m}}=\frac{d^{m}}{d x^{m}} \circ I_{a}^{0}=I_{a}^{-m}
$$

This proves (ii). To show (i), it is enough to differentiate Equation (8) with respect to $a$. One finds

$$
\frac{-1}{\Gamma(s)} f(a)(x-a)^{s-1}
$$

which vanishes for all $f$ continuous on an interval containing $a$ and $x$ only if $s$ is a pole of $\Gamma(s)$, i.e. only for $s$ a nonpositive integer. This completes the proof.

As has been noted, $G$ is generated by real translations $T_{a}$ and the involution $J$. Since $d^{m} / d z^{m}$ is linear, it automatically satisfies equation (2) for $g=T_{a}$. For $g=J$ one can apply the operators $I_{a}^{s}$ as follows:

$$
\left(I_{1}^{s} f\right)\left(y^{-1}\right)=\frac{1}{\Gamma(s)} \int_{1}^{y^{-1}} f(u)\left(y^{-1}-u\right)^{s-1} d u .
$$

Introduce $u=x^{-1}$ :

$$
\begin{aligned}
\left(I_{1}^{s} f\right)\left(y^{-1}\right) & =\frac{(-1)^{s} y^{1-s}}{\Gamma(s)} \int_{1}^{y} f\left(x^{-1}\right) x^{-s-1}(y-x)^{s-1} d x \\
& =(-1)^{s} y^{1-s} I_{1}^{s} y^{-(1+s)} f\left(y^{-1}\right) .
\end{aligned}
$$

Application of Lemma 1 with $s=-m$ yields

$$
\frac{d^{m}}{d\left(y^{-1}\right)^{m}}=(-1)^{m} y^{m+1} \frac{d^{m}}{d y^{m}} \circ y^{m-1},
$$

which becomes, after applying the principle of analytic continuation,

$$
\frac{d^{m}}{d(J z)^{m}}=z^{m+1} \frac{d^{m}}{d z^{m}} \circ z^{m-1}
$$

this is equivalent to equation (7). 
3. Hereafter the operator $d^{m} / d z^{m}$ will be denoted by $L_{z}^{m}$, or $L^{m}$ when it is not necessary to focus attention on the variable. Let $\mathscr{V}^{0}(\Gamma, k, v)$ denote the set of complex valued functions defined on the upper half plane $H$ which satisfy the functional equation for automorphic forms belonging to $\mathscr{V}(\Gamma, k, v)$, which are analytic with the exception of a set of points which is the orbit under $\Gamma$ of a finite set, and which are bounded at the parabolic vertices of $\Gamma$ in terms of a local uniformizing parameter.

Suppose that $f \in \mathscr{V}(\Gamma, k, v)$ and that $k \neq 0$. Then

$$
(f)^{(1-m) / k} \in \mathscr{V}^{0}\left(\Gamma, 1-m, v^{(1-m) / k}\right) \text { for } m>1 .
$$

A calculation shows that

$$
L^{m}\left((f)^{(1-m) / k}\right)=(f)^{(1-(k+1) m) / k} D^{m}(f),
$$

where $D^{m}(f) \in \boldsymbol{R}\left[f, f_{1}, f_{2}, \cdots, f_{m}\right]$. Furthermore, $f$ and $D^{m}(f)$ are relatively prime, and, as a polynomial in $f_{m}, D^{m}(f)$ has the form

$$
D^{m}(f)=\frac{1-m}{k}(f)^{m-1} f_{m}+\text { terms not containing } f_{m} .
$$

Then

$$
D^{m}(f)=(f)^{((k+1) m-1) / k} L^{m}\left((f)^{(1-m) / k}\right) \in \mathscr{V}^{0}\left(\Gamma, m(k+2), v^{m}\right)
$$

for integers $m>1$. Since $D^{m}(f)$ is a polynomial in $f$ and its derivatives, $D^{m}(f)$ is analytic on $H$ and bounded at the parabolic cusps in a local uniformizing parameter, and therefore

$$
D^{m}(f) \in \mathscr{V}\left(\Gamma, m(k+2), v^{m}\right) .
$$

Lemma 2. Suppose $k \neq 0, \Gamma$ a horocyclic group, and $f \in \mathscr{V}(\Gamma, k, v)$, and let $\mathscr{B} \in C\left[f, f_{1}, f_{2}, \cdots, f_{n}\right]$ be an automorphic form for $\Gamma$. Let a typical monomial term of $\mathscr{B}$ be af ${ }^{l_{0}} f_{1}^{l_{1}} \cdots f_{n}^{l_{n}}$ with $a \in C$ and nonnegative integers $l_{\imath}$. Then

$$
l=\sum_{j=0}^{n} l_{j}
$$

and

$$
l^{*}=\sum_{j=0}^{n} j l_{j}
$$

are constant for all monomial terms of $\mathscr{B}$.

This statement and its proof are given by Rankin [3, p. 104]. Under the action of a $\gamma \in \Gamma$, the monomial $a f^{l_{0}} f_{1}^{l_{1}} \ldots f_{n}^{l_{n}}$ is transformed into

$$
a(v(\gamma))^{l}(c z+d)^{k l+2 l^{*}} f^{l_{0}} f_{1}^{l_{1}} \cdots f_{n}^{l_{n}}+\mathscr{R}
$$

where $\mathscr{R}$ contains powers of $(c /(c z+d))$, and $\gamma z=(a z+b) /(c z+d)$. Then a necessary condition that $\mathscr{B}$ be an automorphic form for $\Gamma$ is that the powers of the multi- 
plier and jacobian respectively are constant for all monomial terms of $\mathscr{B}$, i.e. that

$$
l \text { and } k l+2 l^{*}
$$

are constant for all monomials of $\mathscr{B}$.

THEOREM 1. Suppose $k \neq 0, \Gamma$ a horocyclic group, and $f \in \mathscr{V}(\Gamma, k, v)$. Let $l$ and $l^{*}$ be defined by equations (16) and (17). Then every polynomial $\mathscr{B} \in C \quad\left[f, f_{1}, f_{2}, \cdots, f_{m}\right]$ which is an automorphic form for $\Gamma$ is a polynomial in the ring $C\left[f, D^{2}(f), \cdots, D^{m}(f)\right]$ divided by $f^{l-l^{*}}$.

Proof. Rankin's [3] proof works in this situation also. If $f \in \mathscr{V}(\Gamma, k, v)$ and $\mathscr{B} \in C\left[f, f_{1}, \cdots, f_{m}\right]$, then $\mathscr{B}$ can be written as a finite sum of the form

$$
\mathscr{B}=\sum a_{n_{0} n_{1} \ldots n_{m}}(f)^{n_{0}}\left(f_{1}\right)^{n_{1}} \cdots\left(f_{m}\right)^{n_{m}} .
$$

Equation (13) shows that $f_{m}$ can be expressed as a polynomial in $f, D^{m}(f)$, and the $f_{i}, i \leqq i<m$, multiplied by a negative integral power of $f$, as long as $m>1$ and $k \neq 0$. Hence $(f)^{l^{*-l}} \mathscr{B} \in C\left[f, f_{1}, D^{2}(f), \cdots, D^{m}(f)\right]$, by Lemma 2 and equation (13).

Now choose a $\gamma \in \Gamma$ of the form $\gamma z=(a z+b) /(c z+d)$, with $c d \neq 0$. This is possible because $\Gamma$ is horocyclic. We see that

$$
f_{1}(\gamma z)=v(\gamma)(c z+d)^{k+2}\left\{f_{1}(z)+\frac{k c}{c z+d} f(z)\right\}
$$

and we know that

$$
\left(D^{m} f\right)(\gamma z)=v(\gamma)^{m}(c z+d)^{m(k+2)}\left(D^{m} f\right)(z) .
$$

Since $\mathscr{B}$ belongs to some $\mathscr{V}\left(\Gamma, k^{\prime}, v^{\prime}\right)$, then

$$
\begin{aligned}
f^{l^{*}-l}(\gamma z) \mathscr{B}(\gamma z)= & v(\gamma)^{l^{*}-l^{\prime}} v^{\prime}(\gamma)(c z+d)^{k^{\prime}+k\left(l^{*}-l\right)} f^{l^{*}-l}(z) \mathscr{B}(z) \\
= & \sum b_{n_{0} n_{1} \ldots n_{m}} v(\gamma)^{n_{0}+l^{*}-n_{1}}(c z+d)^{k n_{0}+(k+2)\left(l^{*}-l_{1}\right)} \\
& \times\left(\prod_{j=2}^{m}\left(D^{j} f\right)^{n_{j}}\right)\left(v(\gamma)(c z+d)^{k+2}\left\{f_{1}(z)+\frac{k c}{c z+d} f(z)\right)^{n_{1}} .\right.
\end{aligned}
$$

The terms containing $k c /(c z+d)$ must not appear in the final expression. There are distinct transformations $\gamma \in \Gamma$ corresponding to infinitely many distinct functions $c /(c z+d)$, which impose infinitely many conditions on the finite set of coefficients of $\mathscr{B}$, and this is possible only if $b_{n_{0}, n_{1}, \ldots, n_{m}}=0$ if $n_{1}>0$, i.e. only if $f_{1}$ does not appear in $\mathscr{B}$. This completes the proof.

REMARK. In [3], Rankin makes the additional hypothesis that $k$ is not a negative integer, but this is seen to be unnecessary if the $D^{m}(f)$ are used to construct $\mathscr{B}$ rather than the $\psi_{m}(f)$. Otherwise, the proof given above is the same as Rankin's proof of the corresponding theorem for the $\psi_{m}(f)$. 
A comparison of Theorem 1 of [3] with Theorem 1 proved above shows that the set of all automorphic forms for $\Gamma$ that can be expressed as polynomials in $f$ and its derivatives can be equally represented by means of $f$ and the $\psi_{m}(f)$, or by $f$ and the $D^{m}(f)$. It is of interest to determine the relationship of the $\psi_{m}(f)$ to the $D^{m}(f)$.

Proposition 1.

$$
\begin{gathered}
\psi_{2}(f)=\frac{-1}{k^{2} \Gamma(k+1) \Gamma(k+2)} D^{2}(f) . \\
\psi_{3}(f)=\frac{-1}{k^{3} \Gamma(k+1) \Gamma(k+2) \Gamma(k+3)} D^{3}(f) . \\
\text { If } m>3, \text { then } \psi_{m}(f) \text { is not proportional to } D^{m}(f) .
\end{gathered}
$$

Proof. The expression for $\psi_{m}(f)$ given by equation (1) shows that the term containing the highest power of $f_{1}$ appearing in $\psi_{m}(f)$ is

$$
\left(\frac{f_{1}}{\Gamma(k+1)}\right)^{m}
$$

and the term containing $f_{m}$ is

$$
\frac{(-1)^{m-1}(f)^{m-1} f_{m}}{(\Gamma(k))^{m-1} \Gamma(m) \Gamma(k+m)}
$$

The corresponding terms in $D^{m}(f)$ are easily calculated from equation (14); they are

$$
\left[\frac{\prod_{l=0}^{m-1}(1-m-l k)}{k^{m}}\right]\left(f_{1}\right)^{m}
$$

and

$$
\frac{1-m}{k}(f)^{m-1} f_{m}
$$

respectively. Consequently, a necessary condition that $\psi_{m}(f)$ be proportional to $D^{m}(f)$ is

$$
\frac{\left(f_{1}\right)^{m}(\Gamma(k))^{m-1} \Gamma(k+m) \Gamma(m)}{(\Gamma(k+1))^{m}(-1)^{m-1}(f)^{m-1} f_{m}}=\frac{\prod_{l=0}^{m-1}(1-m-l k)\left(f_{1}\right)^{m} \cdot k}{k^{m}(f)^{m-1} f_{m}}
$$

must hold identically in $k$.

It is easy to prove that $m$ must be 2 or 3 if equation (20) holds identically in $k$, and in these cases, a calculation leads to the expressions (i) and (ii) of the proposition, which completes the proof. 
4. The expression for $\psi_{m}(f)$ given by equation (1) and the analogous role played by $\psi_{m}(f)$ and $D^{m}(f)$ in Theorem 1 and Rankin's Theorem 1 [3] hint that there is not likely to be a simple explicit expression for the polynomial $D^{m}(f) \in C\left[f, f_{1}, \cdots, f_{m}\right]$. Nevertheless, it may be of some interest to exhibit an expression for $D^{m}(f)$ similar to that in equation (1), if only for the sake of completeness.

The essence of the matter is the explicit expression of the $m$ th derivative of a power of a function. Because this elementary result does not seem to appear in the standard textbooks, I will record it here.

LeMma 3. Let $h: R \rightarrow R$ be an m-times differentiable function, and let $s \in \boldsymbol{R}$ be a nonzero constant. Set

$$
e_{j, k}=\frac{(k-1) !}{(j-1) !(k-j) !} \frac{d^{j-1}}{d x^{j-1}}\left(\frac{1}{f} \frac{d f}{d x}\right), \quad k \geqq j .
$$

Then

$$
\frac{d^{m}}{d x^{m}}(f(x))^{s}=s^{m} f^{s}(x)\left|\begin{array}{ccc}
e_{1, m}, & e_{2, m}, e_{3, m}, & \cdots e_{m, m} \\
-s^{-1}, e_{1, m-1}, e_{2, m-1}, \cdots & e_{m-1, m-1} \\
0, & -s^{-1}, e_{1, m-2}, \cdots & e_{m-2, m-2} \\
0, & 0, & -s^{-1}, \cdots \\
\vdots & \vdots \\
0, & 0, \cdots & \cdots, 0,-s^{-1}, e_{1,1}
\end{array}\right| .
$$

Remark. Together with the expression

$$
\frac{d^{m}}{d x^{m}}\left(\frac{1}{f} \frac{d f}{d x}\right)=\sum_{j+k=m}\left(\begin{array}{c}
m \\
j
\end{array}\right)_{f_{j+1}}\left(f^{-1}\right)_{k}
$$

and an explicit expression for $\left(f^{-1}\right)_{k}$ which is easily found from the set of equations

$$
0=\sum_{j+k=m}\left(\begin{array}{c}
m \\
j
\end{array}\right) f_{j}\left(f^{-1}\right)_{k}, \quad m=1,2, \cdots,
$$

Lemma 3 leads to a complicated explicit expression for $D^{m}(f)$.

5. Some elementary properties of the nonlinear operators $D^{m}$ will be useful later.

Lemma 4. (i) If $c \in C$, then $D^{m}(c f)=c^{m} D^{m}(f)$;

(ii) If $s \in C$ is a nonzero constant, and $f \in \mathscr{V}(\Gamma, k, v)$, then

$$
D^{m}\left(f^{s}\right)=f^{(s-1) m} D^{m}(f) .
$$


Both identities follow from equation (14), which defines $D^{m}(f)$. For (ii) it is necessary to note that $f^{s} \in \mathscr{V}\left(\Gamma, s k, v^{s}\right)$.

Once again suppose that $f \in \mathscr{V}(\Gamma, k, v)$ and in addition that $f$ has a Fourier expansion of the form

$$
f(z)=\sum_{j \geqq 0} a(j) \exp (2 \pi i j z / \lambda)
$$

with $\lambda$ a positive real number. Define the $\operatorname{order}$ of $f$, written $\operatorname{ord}(f)$, as the greatest integer $r \geqq 0$ such that $a(j)=0$ for $j<r$. The following property of the $D^{m}$ is most helpful.

Proposition 2. (i) For every $m \geqq 2, \operatorname{ord}\left(D^{m}(f)\right)>0$.

(ii) If $\operatorname{ord}(f)>0$, then $\left.\operatorname{ord}\left(D^{m}\right)(f)\right) \geqq m$ ord $(f)$.

Proof. Each monomial term in the expression for $D^{m}(f) \in \boldsymbol{R}\left[f, f_{1}, \cdots, f_{m}\right]$ is, apart from irrelevant constant factors, a product of $m$ functions, each of which is a member of the set $\left\{f, f_{1}, \cdots, f_{m}\right\}$, subject to the condition that at least one of the derivatives $f_{\imath}, 1 \leqq i \leqq m$, appears. Hence the Fourier expansion of $D^{m}(f)$ cannot contain a nonzero constant term, which proves (i).

The second assertion is evident because, according to the hypothesis, ord $(f)>0$, and so $\operatorname{ord}\left(f_{i}\right)=\operatorname{ord}(f)$ for each derivative. Now the monomial terms of $D^{m}(f)$ have order equal to the sum of the orders of their factors, hence equal to $m \operatorname{ord}(f)$. The linear combination of these monomials which is $D^{m}(f)$ cannot decrease order, which completes the proof.

REMARK. If a suitably chosen fundamental domain in $H$ for the horocyclic group $\Gamma$ has one cusp, located at infinity, then the proposition asserts that $D^{m}(f)$ is a cusp form for every integer $m \geqq 2$ and every $\Gamma$-automorphic form $f$. In particular, this is the case for the classical modular group.

6. Let $\Gamma$ be a discrete group acting on the upper half plane $H$. It is well known that a necessary and sufficient condition that $H / \Gamma$ is compactifiable to a compact Riemann surface $\mathscr{R}$ is that the area of a suitably chosen fundamental domain for $\Gamma$ in $H$ be finite with respect to the invariant metric.

According to a famous theorem, any two meromorphic functions $f, g$ on $\mathscr{R}$ are algebraically dependent over $\boldsymbol{C}$. These facts make it possible to prove that every reasonably well behaved automorphic form or automorphic function satisfies an algebraic differential equation. More precisely:

THEOREM 2. If $\Gamma$ is a discrete group acting on $H$ with a fundamental domain of finite invariant area, and if $f \in \mathscr{V}(\Gamma, k, v)$ is an analytic automorphic form with a multiplier system $v$ satisfying $v \equiv 1$ for some positive integer $s$, and if moreover $k s$ is a positive integer, then there is a polynomial $\mathscr{B} \in C\left[x_{1}, x_{2}, x_{3}\right]$ such that

$$
\mathscr{B}\left(f, D^{2}(f), D^{3}(f)\right)=0 \quad \text { on } H,
$$


i.e., such that $f$ satisfies an algebraic differential equation of order less than 4.

Proof. The hypotheses show that $f^{s} \in \mathscr{V}(\Gamma, k s, 1)$, and equation (15) states that $\left(D^{2}\left(f^{s}\right)\right)^{3}$ and $\left(D^{3}\left(f^{s}\right)\right)^{2}$ belong to $\mathscr{V}(\Gamma, 6(k s+2), 1)$.

If $l$ is the least common multiple of $k s$ and $6(k s+2)$, then

$$
f^{l / k},\left(D^{2}\left(f^{s}\right)\right)^{l / 3(k s+2)} \text {, and }\left(D^{3}\left(f^{s}\right)\right)^{l / 2(k s+2)}
$$

all have the same dimension and multiplier system. Then $\left(D^{3}\left(f^{s}\right)\right)^{l / 2(k s+2)} / f^{l / k}$ and $\left(D^{2}\left(f^{s}\right)\right)^{l / 3(k s+2)} / f^{l / k}$ are meromorphic functions on $H / \Gamma$ and are therefore algebraically dependent. Clearing the denominator of the rational expression which exhibits this algebraic dependence produces a polynomial equation $\mathscr{B}^{*}=0$ satisfied by the three forms $(*)$.

Now it is only necessary to recall the second assertion of Lemma 4, which contains the identities

$$
D^{2}\left(f^{s}\right)=f^{2 s-2} D^{2}(f) \text { and } D^{3}\left(f^{s}\right)=f^{3 s-3} D^{3}(f),
$$

in order to obtain from $\mathscr{B}^{*}$ a polynomial $\mathscr{B}$ such that $\mathscr{B}\left(f, D^{2}(f), D^{3}(f)\right)=0$ on $H$. This completes the proof.

REMARK. For a discrete group $\Gamma$ satisfying the conditions in the theorem, and an arbitrary $f \in \mathscr{V}(\Gamma, k, v)$, it follows that $f$ satisfies an algebraic differential equation of order less than 5. For let $m>3$. Then $D^{m}(f) /\left(D^{2}(f)\right)^{r}\left(D^{3}(f)\right)^{s}$ and $\left(D^{3}(f)\right)^{2} /\left(D^{2}(f)\right)^{3}$ are algebraically dependent if $2 r+3 s=m$ with $r$ and $s$ nonnegative integers. This is so if $m=4$, which proves the remark. The fact that $\psi_{m}, \psi_{2}$, and $\psi_{3}$ are algebraically dependent was noted in [3].

COROLlaRY. With the hypotheses of the theorem for $\Gamma$, every $\Gamma$-automorphic function satisfies an algebraic differential equation of order less than 4.

Proof. An $\Gamma$-automorphic function is a meromorphic function defined on $H$ and invariant under $\Gamma$ which satisfies suitable growth conditions at the cusps. Let $f$ be such a function. Then $f_{1} \in \mathscr{V} 0(\Gamma, 2,1)$ and $D^{2}\left(f_{1}\right) \in \mathscr{V} 0(\Gamma, 8,1)$. Hence $D^{2}\left(f_{1}\right) /\left(f_{1}\right)^{4}$ and $f$ are two $\Gamma$-automorphic functions. Since the area of a fundamental domain for $\Gamma$ is finite, these functions are algebraically dependent. As in the proof of the Theorem, this leads to a polynomial in three indeterminates which vanishes on the triple $\left(f, f_{1}, D^{2}\left(f_{1}\right)\right)$, which completes this proof.

REMARK. The operator $f \rightarrow D^{2}\left(f_{1}\right) /\left(f_{1}\right)^{4}$ takes automorphic functions onto automorphic functions, and is, as one might suspect, related to the Schwarzian derivative,

$$
S(f)=\frac{f_{1} f_{3}-(3 / 2)\left(f_{2}\right)^{2}}{\left(f_{1}\right)^{2}} .
$$

In fact, $D^{2}\left(f_{1}\right) /\left(f_{1}\right)^{4}=\frac{1}{2} S(f) /\left(f_{1}\right)^{2}$. It is obvious than an infinite number of differential operators taking automorphic functions onto automorphic functions can 
be constructed from $f, f_{1}$ and the $D^{m}(f)$, and it is not hard to show that all operators with this property which are rational functions of $f$ and its derivatives have the form $\mathscr{B}_{1} / \mathscr{B}_{2}$ where $\mathscr{B}_{i}$ is a polynomial in $f, f_{1}$ and the $D^{m}\left(f_{1}\right), i=1,2$.

7. In [3] Rankin gave a homogeneous fourth order differential equation satisfied by the normalized cusp form of dimension -12 for the classical modular group,

$$
\Delta(z)=e^{2 \pi i z}\left\{\prod_{n=1}^{\infty}(1-\exp (2 \pi i n z)\}^{24} ;\right.
$$

he also remarked that a nonhomogeneous third order equation could be found.

Proposition 3. $27\left(D^{3} \Delta\right)^{2}-1728\left(D^{2} \Delta\right)^{3}-64 \pi^{6} \Delta^{7}=0$. (Note that $1728=$ (27)(64).)

Proof. Proposition 2 and the classical theorem describing generators of the graded ring of modular forms shows that there are constants $c_{1}$ and $c_{2}$ such that

$$
D^{2} \Delta=c_{1} G_{4} \Delta^{2} \text { and } D^{3} \Delta=c_{2} G_{6} \Delta^{3},
$$

where the normalized Eisenstein series $G_{k}$ is defined by

$$
G_{k}=1+\frac{(-1)^{k / 2}(2 \pi)^{k}}{(k-1) ! \zeta(k)} \sum_{n=1}^{\infty} \sigma_{k-1}(n) \exp (2 \pi i n z),
$$

$\sigma_{l}(n)=\Sigma_{d / n ; d>0} d^{l}, \zeta$ is the Riemann Zeta function, and $k$ is an even integer greater than 2 .

An easy computation shows that

$$
D^{2} \Delta=\frac{(2 \pi i)^{2}}{(12)^{2}} G_{4} \Delta^{2},
$$

and

$$
D^{3} \Delta=-\frac{(2 \pi i)^{3}}{(6)^{3}} G_{6} \Delta^{3} .
$$

The proposition follows upon application of the classical identity

$$
(12)^{3} \Delta=\left(G_{4}\right)^{3}-\left(G_{6}\right)^{2}
$$

to eliminate $G_{4}$ and $G_{6}$ from equations (24) and (25).

According to Theorem 2, $G_{4}$ and $G_{6}$ also satisfy algebraic differential equations of order less than 4 . This is particularly interesting because these forms generate the graded ring of modular forms. The explicit equation for $G_{4}$ is not hard to find:

Proposition 4. 5( $\left.D^{3} G_{4}\right)^{2}-576\left(D^{2} G_{4}\right)^{3}+80 \pi^{2}\left(G_{4}\right)^{3}\left(D^{2} G_{4}\right)^{2}=0$. 
Proof. Each monomial belongs to $\mathscr{V}(\Gamma, 36,1)$, so that a linear combination with order greater than 3 must vanish identically. Calculation shows that

$$
D^{2} G_{4}=-(2 \pi i)^{2}(60) \Delta
$$

and

$$
D^{3} G_{4}=-(2 \pi i)^{3}(120) G_{6} \Delta .
$$

Then a suitable linear combination of $\left(D^{3} G_{4}\right)^{2}$ and $\left(G_{4}\right)^{3}\left(D^{2} G_{4}\right)^{2}$ is proportional to $\Delta^{3}$ (by equation (26)), and $\Delta^{3}$ can be expressed in terms of $\left(D^{2} G_{4}\right)$ by equation (27).

A third order differential equation satisfied by $G_{6}$ can be found by looking at the products of $G_{6}, D^{2} G_{6}$, and $D^{3} G_{6}$ which have dimension -144 , but the calculations are too distasteful to be performed without a compelling reason.

In [6] we gave the differential equation of order 3 for the theta function

$$
\theta(z)=\sum_{n} \exp \left(i \pi n^{2} z\right)
$$

which is an automorphic form of dimension $-\frac{1}{2}$ with respect to the group generated by $z \rightarrow z+2$ and $z \rightarrow-z^{-1}$. The notation in that note differs slightly from the one used here; in the present notation, the equation is:

Proposition 5. $\left(D^{3} \theta\right)^{2}-64\left(D^{2} \theta\right)^{3}+4 \pi^{2} \theta^{10}\left(D^{2} \theta\right)^{2}=0$.

8. In this section some identities for the Fourier coefficients of $G_{4}, G_{6}$ and $\Delta$ are given. Put $\Delta(z)=\Sigma_{1}^{\infty} \tau(n) \exp (2 \pi i n z)$; then the $\tau(n)$ are Ramanujan's numbers. Equation(22)shows that they are integers. As usual, put $\sigma_{l}(n)=\Sigma_{d / n ; d>0} d^{l}$.

Proposition 6.

$$
\tau(n)=n^{2} \sigma_{7}(n)-540 \sum_{j=1}^{n-1} j(n-j) \sigma_{3}(j) \sigma_{3}(n-j)
$$

(ii) $\tau(n)+240 \sum_{j=1}^{n-1} \tau(j) \sigma_{3}(n-j)=n^{2} \sigma_{5}(n)+12 \sum_{j=1}^{n-1}\left(91 j^{2}-42 n j\right) \sigma_{5}(j) \sigma_{5}(n-j)$;

(iii) $240 \sum_{j=1}^{n-1} \sum_{t=1}^{n-1} \sigma_{3}(j) \tau(t) \tau(n-j-t)=\sum_{j=1}^{n-1}\left(13 j n-25 j^{2}-1\right) \tau(j) \tau(n-j)$.

Proof. Equation (27) is equivalent to the Fourier coefficient identity

$$
\tau(n)=n^{2} \sigma_{3}(n)+60 \sum_{j=1}^{n-1}\left(4 n^{2}-13 n j+9 j^{2}\right) \sigma_{3}(j) \sigma_{3}(n-j),
$$

which appears incorrectly in [7], equation (53). The more symmetrical form (i) is obtained by using the classical identity

$$
\sigma_{7}(n)=\sigma_{3}(n)+120 \sum_{j=1}^{n-1} \sigma_{3}(j) \sigma_{3}(n-j) \text {. }
$$


Identity (ii) is equivalent to equation (28), and (iii) is equivalent to equation (24).

9. We have already made use of the classical fact that $G_{4}$ and $G_{6}$ generate the graded ring $\bigoplus_{k} \mathscr{V}\left(\Gamma_{m}, k, 1\right)$ of automorphic forms for the classical modular group $\Gamma_{m}(=\operatorname{SL}(2, Z)$ modulo its center). In this section we will make use of the differential operators $D^{m}$ to show that the generators of $\bigoplus_{k} \mathscr{V}\left(\Gamma_{m}, k, 1\right)$ can be expressed by means of the $D^{m}$ and either one of $G_{4}$ or $\Delta$. This peculiar result is of interest because the differential operators $D^{m}$ depend on the full group of analytic automorphisms of $H$, i.e. only on $\operatorname{SL}(2, R)$ modulo its center, and not upon the particular nature of any discrete subgroup. From this remark it follows that another way of stating the result is that the generators of $\bigoplus_{k} \mathscr{V}\left(\Gamma_{m}, k, 1\right)$ are known if either of $G_{4}$ or $\Delta$ is known, and if certain geometric properties of the upper half plane are known.

THEOREM 3. Each of the following pairs generates $\bigoplus_{k} \mathscr{V}\left(\Gamma_{m}, k, 1\right)$ :

(i) $G_{4},\left(D^{3} G_{4}\right) / D^{2} G_{4}$;

(ii) $D^{2} \Delta / \Delta^{2}, D^{3} \Delta / \Delta^{3}$.

Proof. Equations (27) and (28) show that $D^{3} G_{4} / D^{2} G_{4}$ differs from $G_{6}$ by a nonzero constant factor; this proves (i). Similarly, equations (24) and (25) prove that $D^{2} \Delta / \Delta^{2}$ is a multiple of $G_{4}$, and $D^{3} \Delta / \Delta^{3}$ is a multiple of $G_{6}$, which proves (ii), and the theorem.

REMARK 1. It does not seem to be possible to express $G_{4}$ as a rational function of $G_{6}$ and the $D^{m} G_{6}$. However, it is easy to see that there is a constant $c$ such that

$$
\frac{D^{3}\left(D^{2} G_{6}\right)}{G_{6}}-c\left(D^{2} G_{6}\right)^{3}
$$

is a constant multiple of $\left(G_{4}\right)^{4}$.

REMARK 2. Theorem 3 is not an isolated fact. A similar result holds for the graded ring of Siegel modular forms of genus 2, which is known to have four generators. This suggests the conjecture that the graded ring of automorphic forms for a discrete group acting on the tube over a domain of positivity (i.e. the set $\mathfrak{U}+i \exp \mathfrak{A}$, where $\mathfrak{A}$ is a compact real Jordan algebra) is generated by rational functions of some one automorphic form $f$ and certain explicitly known differential operators acting on $f$, if the discrete group has one cusp at infinity.

One can also conjecture that the normalized cusp form of greatest dimension (i.e. least "weight") can be used as the form $f$. This is the case for the classical modular group $(f=\Delta)$ and for the Siegel modular group of genus $2(f=$ cusp form of dimension -10$)$.

\section{BIBLIOGRAPHY}

1. G. Bol, Invarianten linearer Differentialgleichungen, Abh. Math. Sem. Univ. Hamburg 16 (1949), no. 3, 1-28.

2. H. Petersson, Konstruktion der Modulformen u.s.w., S.-B. Heidelberger Akad. Wiss. Math.-Nat. Kl. (1950), 417-494. 
3. R. A. Rankin, The construction of automorphic forms from the derivatives of a given form, J. Soc. Indust. Appl. Math. 20 (1956), 103-116.

4. - The construction of automorphic forms from the derivatives of given forms, Michigan Math. J. 4 (1957), 181-186.

5. M. Riesz, L'integrale de Riemann-Liouville et le probleme de Cauchy, Acta Math. 81 (1949), 1-223.

6. H. L. Resnikoff, $A$ differential equation for the theta function, Proc. Nat. Acad. Sci. U.S.A. 53 (1965), 692-693.

7. B. van der Pol, On a non-linear partial differential equation satisfied by the logarithm of the Jacobian theta-functions, with arithmetical applications. I, II, Indag. Math. 13(1951), 261-284.

The INSTITUTE FOR AdVANCEd STUdy,

Princeton, NeW Jersey 